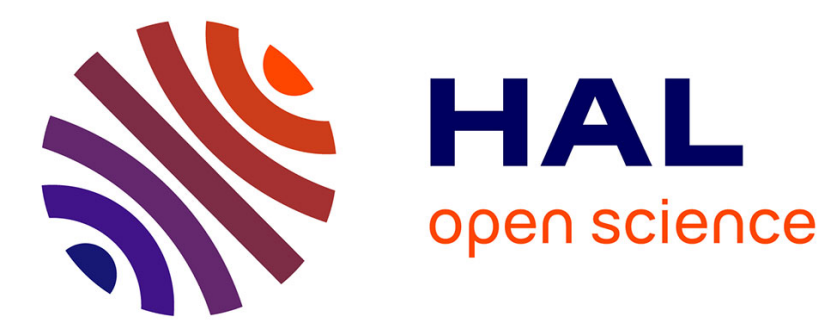

\title{
Evidence for a Peierls transition in the blue bronzes K0.30MoO 3 and Rb0.30MoO3
}

\author{
J.P. Pouget, S. Kagoshima, C. Schlenker, J. Marcus
}

\section{To cite this version:}

J.P. Pouget, S. Kagoshima, C. Schlenker, J. Marcus. Evidence for a Peierls transition in the blue bronzes K0.30MoO 3 and Rb0.30MoO3. Journal de Physique Lettres, 1983, 44 (3), pp.113-120. 10.1051/jphyslet:01983004403011300 . jpa-00232150

\section{HAL Id: jpa-00232150 https://hal.science/jpa-00232150}

Submitted on 1 Jan 1983

HAL is a multi-disciplinary open access archive for the deposit and dissemination of scientific research documents, whether they are published or not. The documents may come from teaching and research institutions in France or abroad, or from public or private research centers.
L'archive ouverte pluridisciplinaire HAL, est destinée au dépôt et à la diffusion de documents scientifiques de niveau recherche, publiés ou non, émanant des établissements d'enseignement et de recherche français ou étrangers, des laboratoires publics ou privés. 
Classification

Physics Abstracts

$61.10-61.60-64.70 \mathrm{~K}-72.20$

\title{
Evidence for a Peierls transition in the blue bronzes $\mathrm{K}_{0.30} \mathrm{MoO}_{3}$ and $\mathrm{Rb}_{0.30} \mathrm{MoO}_{3}$
}

\author{
J. P. Pouget, S. Kagoshima \\ Laboratoire de Physique des Solides (*), Université Paris-Sud, 91405 Orsay, France \\ C. Schlenker and J. Marcus \\ Groupe des Transitions de Phases, C.N.R.S., B.P. 166, 38042 Grenoble Cedex, France
}

(Reçu le 10 novembre 1982, accepté le 15 décembre 1982)

\begin{abstract}
Résumé. - Des études de diffusion de rayons $\mathrm{X}$ montrent que la transition métal-isolant observée à $T_{\mathrm{c}}=180 \mathrm{~K}$ sur les bronzes bleus $\mathrm{K}_{0,30} \mathrm{MoO}_{3}$ et $\mathrm{Rb}_{0,30} \mathrm{MoO}_{3}$, composés quasi unidimensionnels, est accompagnée d'une distorsion périodique de réseau. Une analyse de la diffusion observée audessus de $T_{\mathrm{c}}$, montre que l'instabilité de réseau concerne principalement les octaèdres $\mathrm{MoO}_{6}$. L'anisotropie de la résistivité est compatible avec une bande de conduction quasi unidimensionnelle construite sur les orbitales hybridées $4 d$ des molybdènes et $p_{\pi}$ des oxygènes. Les résultats des études structurales joints aux propriétés de transport confirment que la transition de phase des bronzes bleus est de type Peierls.

Abstract. - X-ray diffuse scattering studies of the quasi one-dimensional blue bronzes $\mathrm{K}_{0.30} \mathrm{MoO}_{3}$ and $\mathrm{Rb}_{0.30} \mathrm{MoO}_{3}$ show that the metal insulator phase transition observed at $T_{\mathrm{c}}=180 \mathrm{~K}$ is accompanied by a periodic lattice distortion. Analysis of the diffuse scattering observed well above $T_{\mathrm{c}}$ shows that the lattice instability involves mainly $\mathrm{MoO}_{6}$ octahedra. The anisotropy of the electrical resistivity is consistent with a quasi one-dimensional conduction band built on hybridized molybdenum $4 \mathrm{~d}$ and oxygen $\mathrm{p}_{\pi}$ orbitals. Together with the electrical properties, the structural results corroborate that the phase transition of the blue bronzes can be viewed as a Peierls distortion.
\end{abstract}

1. Introduction. - The so-called blue bronzes $\mathrm{K}_{0.30} \mathrm{MoO}_{3}$ and $\mathrm{Rb}_{0.30} \mathrm{MoO}_{3}$ belong to the class of the ternary transition metal oxides $\mathrm{M}_{x} \mathrm{TO}_{m}$ where $\mathrm{T}$ is a transition metal and $\mathrm{M}$ an alkaline metal [1]. In these compounds, the alkaline metal usually donates its outer electron to the transition metal and thus fills partially the $\mathrm{d}$ states which are often unoccupied in the oxide $\mathrm{TO}_{m}$. The molybdenum bronzes are intermediate between the metallic tungsten bronzes and the vanadium bronzes where d electrons are more localized. While the red bronze $\mathrm{K}_{0.33} \mathrm{MoO}_{3}$ is a semiconductor at all temperatures [2] and the purple bronze $\mathrm{K}_{0.9} \mathrm{Mo}_{6} \mathrm{O}_{17}$ is a two-dimensional metal exhibiting probably a charge density wave (C.D.W.) driven phase transition at $120 \mathrm{~K}$ [3], the blue bronze

(*) Laboratoire associé au C.N.R.S. 
$\mathrm{K}_{0.30} \mathrm{MoO}_{3}$ exhibits at room temperature quasi one-dimensional (1D) metallic properties and a metal-semiconductor phase transition at $180 \mathrm{~K}$ [4-6]. By analogy with the properties of the organic charge transfer salts such as TTF-TCNQ and the Krogmann salts such as $\mathrm{K}_{2} \mathrm{Pt}(\mathrm{CN})_{4} \mathrm{Br}_{0.3} 3 \mathrm{H}_{2} \mathrm{O}(\mathrm{KCP})$ showing similar electronic anisotropies and undergoing at low temperature structural instabilities related to the formation of C.D.W. [7], it has been suggested that the metal insulator phase transition of the blue bronze might be of the Peierls type.

Structural evidences of an electronic instability towards the formation of C.D.W. in the blue bronzes $\mathrm{K}_{0.30} \mathrm{MoO}_{3}$ and $\mathrm{Rb}_{0.30} \mathrm{MoO}_{3}$ are the purpose of this letter.

$\mathrm{K}_{0.30} \mathrm{MoO}_{3}$ belongs to the monoclinic space group $\mathrm{C} 2 / \mathrm{m}$ [8] with twenty formulae per unit cell. The crystallographic structure is built with infinite sheets of $\mathrm{MoO}_{6}$ octahedra separated by the $\mathrm{K}$ ions. The $\mathrm{MoO}_{6}$ layers consist of clusters of octahedra linked by corners in the [010] and [102] directions; the cluster itself contains $10 \mathrm{MoO}_{6}$ octahedra sharing edges. Packing of clusters is steplike along the [102] direction and in column along the [010] direction. The structure can then be viewed as containing infinite chains of $\mathrm{MoO}_{6}$ octahedra sharing corners along the monoclinic $b$ direction. There are three different Mo sites per cluster; only two of them $(\mathrm{Mo}(2)$ and $M o(3)$ in Ref. [8]) are involved in these infinite chains. Chenevas, Ghedira and Marezio have established that $80 \%$ of the $4 d$ electronic density is found on the $M o(2)$ and $M o(3)$ sites [9]. Above $180 \mathrm{~K}, \mathrm{~K}_{0.30} \mathrm{MoO}_{3}$ is a quasi one-dimensional metal as shown by the anisotropy of the electrical resistivity and by the observation of a metallic-like plasma edge in the optical reflectivity when the light is polarized parallel to the $b$-monoclinic axis and its absence when the light is polarized perpendicular to $b[5,6]$. It has been proposed previously that the conduction band is built on hybridized $\mathrm{p}_{\pi}$-d orbitals involving the $\mathrm{Mo}(2) \mathrm{O}_{6}$ and $\mathrm{Mo}(3) \mathrm{O}_{6}$ octahedra only [6]. One should also point out that, if the stoichiometry corresponds exactly to the formula $\mathrm{M}_{0.30} \mathrm{MoO}_{3}$, all crystallographic sites are occupied and therefore no crystallographic disorder is expected.

The transport and structural properties of $\mathrm{Rb}_{0.30} \mathrm{MoO}_{3}$ are completely similar to those of $\mathrm{K}_{0.30} \mathrm{MoO}_{3}[5,9]$.

2. Experimental techniques. - Single crystals of the blue bronzes have been obtained by the electrolytic reduction of a $\mathrm{M}_{2} \mathrm{MoO}_{4}-\mathrm{MoO}_{3}(\mathrm{M}=\mathrm{K}, \mathrm{Rb})$ melt. Crystals are platelets of typical size $5 \times 2 \times 1 \mathrm{~mm}^{3}$ parallel to the $(\overline{201})$ cleavage plane, with $b$ as the long direction. Structural refinements show that the chemical formulae are close to $\mathrm{K}_{0.30} \mathrm{MoO}_{3}$ and $\mathrm{Rb}_{0.30} \mathrm{MoO}_{3}$ [9] (and not $\mathrm{Rb}_{0.23} \mathrm{MoO}_{3}$ as proposed in Ref. [10]).

The electrical conductivity has been measured between $4.2 \mathrm{~K}$ and $300 \mathrm{~K}$ by the four point technique using a gold paste on freshly cleaved crystals.

The X-ray diffuse scattering study was performed using the " monochromatic Laue ", oscillating crystal and Weissenberg photographic techniques. The $\mathrm{CuK}_{\alpha}$ radiation $(1.542 \AA)$ was obtained by reflection, on a doubly bent graphite monochromator (002 reflection), of the X-ray beam produced by a $12 \mathrm{KW}$ Rigaku rotating anode generator. The crystals were cooled by a regulated nitrogen flux and temperatures, between $295 \mathrm{~K}$ and $110 \mathrm{~K}$, were measured by a thermocouple glued on the sample.

X-ray patterns were analysed using a Joyce-Lœble microdensitometer, and the half-width at half maximum of the diffuse scattering, shown in figure 4 , was obtained from such reading.

3. Results. - Figure 1 shows a typical temperature dependence of the electrical resistivity of $\mathrm{Rb}_{0.30} \mathrm{MoO}_{3}$ measured along three orthogonal crystallographic directions. Similar results have been obtained with $\mathrm{K}_{0.30} \mathrm{MoO}_{3}$ [5]. $\mathrm{Rb}_{0.30} \mathrm{MoO}_{3}$ shows a metal-semiconductor phase transition at the same critical temperature, $T_{\mathrm{c}}=180 \mathrm{~K}$, as $\mathrm{K}_{0.30} \mathrm{MoO}_{3}$ [5]. In both materials the highest conductivity is measured along the chain-like $b$ direction, the intermediate conductivity along 


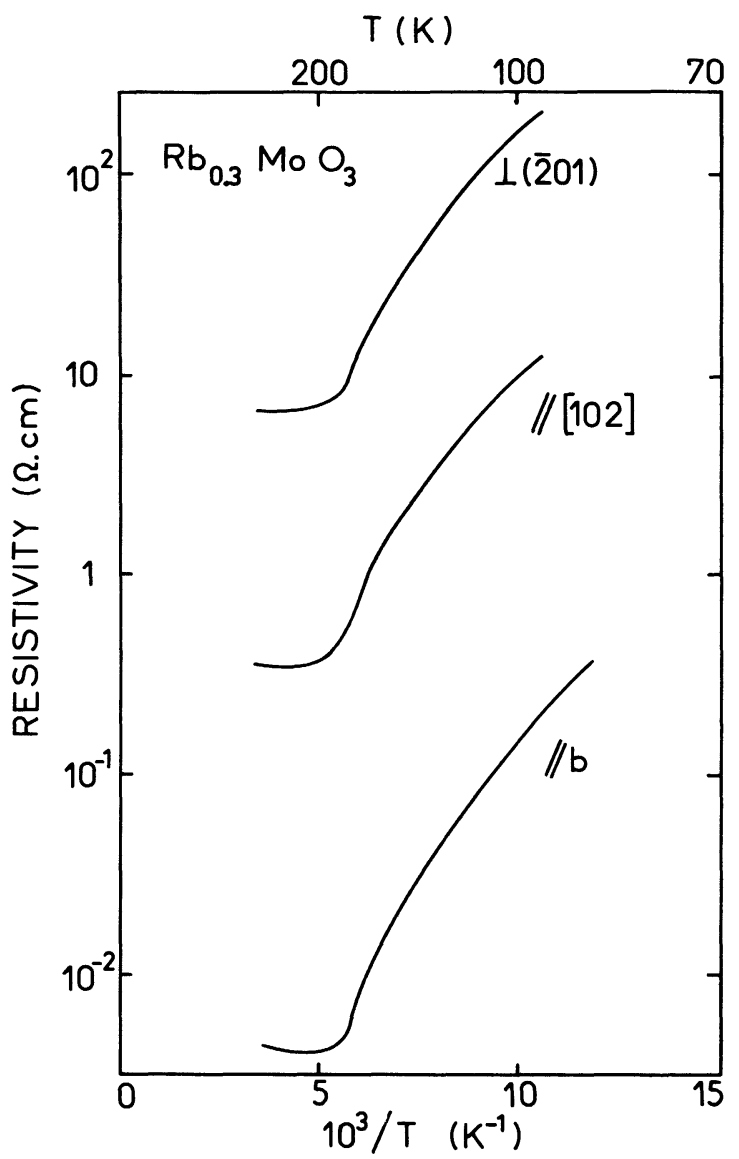

Fig. 1. - Electrical resistivity (logarithmic scale) of $\mathrm{Rb}_{0.30} \mathrm{MoO}_{3}$ versus reciprocal temperature, measured along b, along [102] and perpendicular to the (201) plane.

the [102] direction and the smallest conductivity in the direction perpendicular to the infinite layers $(\overline{2} 01)$ of oxygen octahedra, as expected from the structural anisotropy. At room temperature the ratio of resistivities measured in the (201) plane, $\rho[102] / \rho_{b}$, is $\sim 76$ for $\mathrm{Rb}_{0.30} \mathrm{MoO}_{3}$ and $\sim 33$ for $\mathrm{K}_{0.30} \mathrm{MoO}_{3}$ and the ratio between the smallest and the highest resistivities, $\rho / \rho_{b}$, is $\sim 1450$ in $\mathrm{Rb}_{0.30} \mathrm{MoO}_{3}$ and $\sim 580$ in $\mathrm{K}_{0.30} \mathrm{MoO}_{3}$. These anisotropies are comparable to those found in the quasi one-dimensional conductor HMTSF-TCNQ [11].

X-ray evidence of a C.D.W. instability leading to a periodic lattice distortion below $T_{\mathrm{c}}$ in $\mathrm{K}_{0.30} \mathrm{MoO}_{3}$ and $\mathrm{Rb}_{0.30} \mathrm{MoO}_{3}$ are well summarized by the two X-ray patterns shown in figure 2 . At room temperature (R.T.), the "monochromatic Laue "X-ray pattern of figure $2 a$ shows clearly thin diffuse segments perpendicular to the $b$ direction and situated at $\pm q_{b} b^{*}$ from layers of Bragg reflections with odd $K$ indices; with for both compounds the same incommensurate wave vector $q_{b}=0.28 \pm 0.01 b^{*}$. Moreover figure $2 a$ shows that this scattering does not correspond to diffuse sheets in the reciprocal space. More clearly X-ray Weissenberg $\left[h, 1 \pm q_{b}, l\right]$ reciprocal planes reveal broad diffuse streaks parallel to the $2 \mathbf{a}^{*}-\mathbf{c}^{*}$ reciprocal direction (perpendicular to the octahedra layers), as schematically shown in figure $3 a$. Weissenberg X-ray patterns taken with $\mathrm{K}_{0.30} \mathrm{MoO}_{3}$ and $\mathrm{Rb}_{0.30} \mathrm{MoO}_{3}$ show a similar wave vector dependence of the diffuse scattering. 


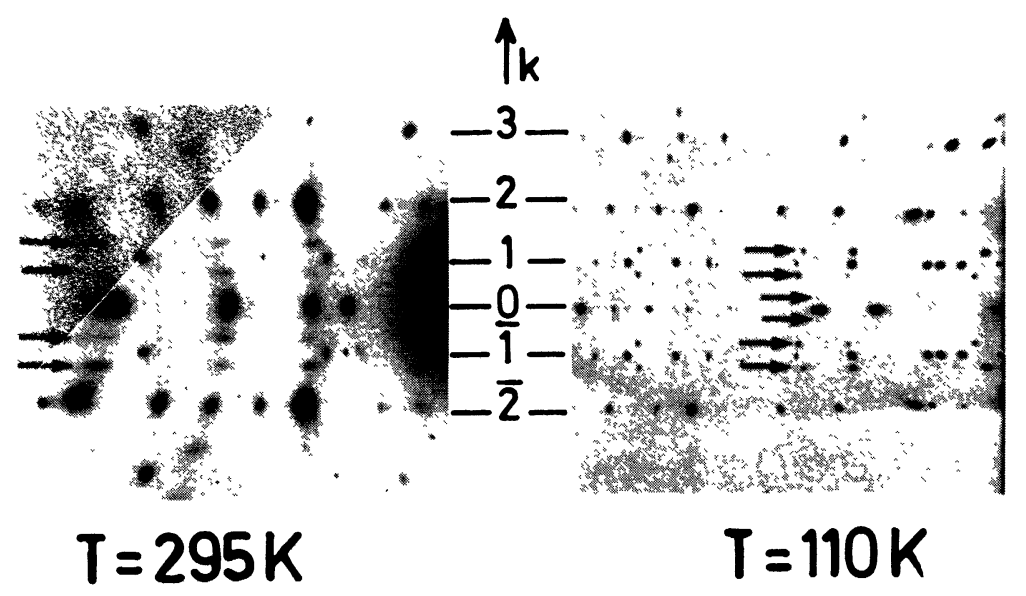

a)

b)

Fig. 2. - « Monochromatic Laue » $(a)$ and oscillating crystal $\left(\theta \sim 10^{\circ}\right)(b)$ X-ray patterns from $\mathrm{Rb}_{0.30} \mathrm{MoO}_{3}$ at $295 \mathrm{~K}$ and $\mathrm{K}_{0.30} \mathrm{MoO}_{3}$ at $110 \mathrm{~K}$ respectively. Arrows point to the diffuse elongated platelets in $(a)$ and to satellite reflections in $(b)$. On both patterns the $\mathbf{b}^{*}$ direction is vertical. On the X-ray pattern $(a)$, note the broad diffuse lines of weaker intensity directed along $b^{*}$ and containing the diffuse platelets and the main Bragg reflections with even $K$ indices. Similar X-ray patterns were obtained from $\mathrm{K}_{0.30} \mathrm{MoO}_{3}$ at $295 \mathrm{~K}$ and $\mathrm{Rb}_{0.30} \mathrm{MoO}_{3}$ at $110 \mathrm{~K}$.

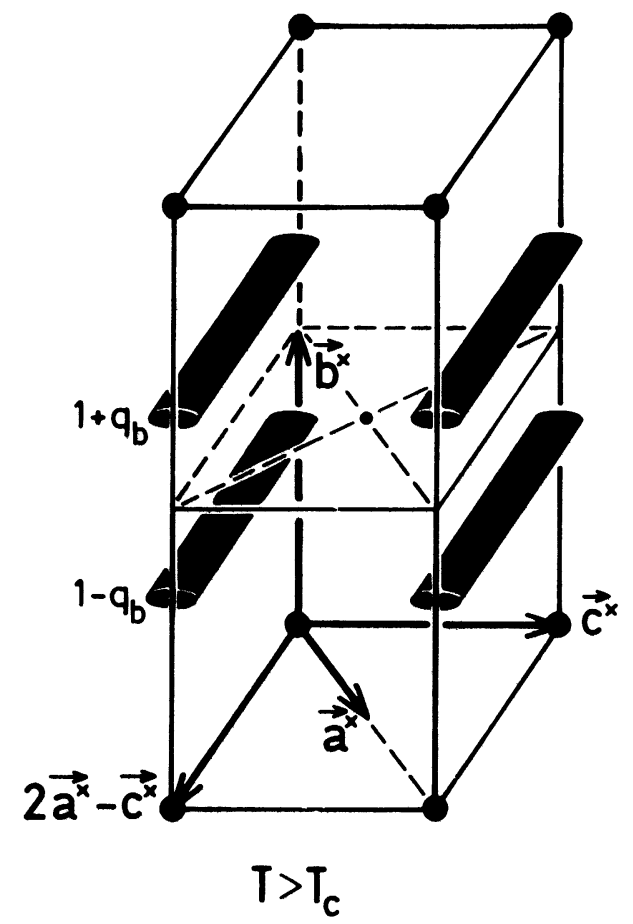

a)

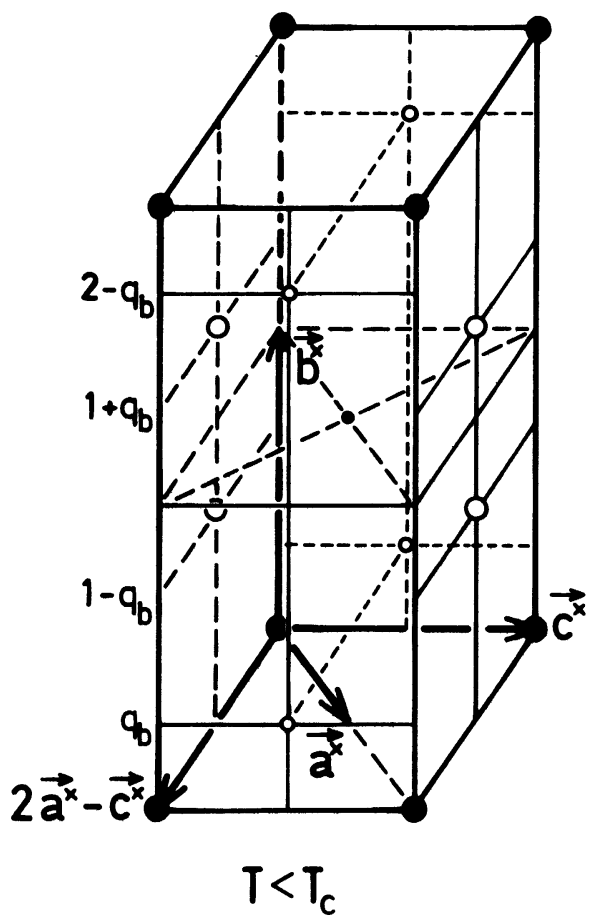

b)

Fig. 3. - Schematic representation of the position in reciprocal space of diffuse platelets for $T>T_{\mathrm{c}}(a)$ and satellites reflections for $T<T_{\mathrm{c}}(b)$. Solid circles represent the main Bragg reflections and open circles represent the satellite reflections. Reciprocal directions used correspond to those of reference [8]. 
A further inspection of the X-ray pattern of figure $2 a$ shows that the diffuse segments correspond to a strengthening at $\pm\left(1-q_{b}\right) \mathbf{b}^{*}= \pm 0.72 \mathrm{~b}^{*}$ of the intensity of an anisotropic thermal diffuse scattering surrounding Bragg reflections with even $H$ and $K$ indices. This thermal scattering also appears under the form of streaks directed in the $2 \mathbf{a}^{*}-\mathbf{c}^{*}$ direction in the $[h, 0, l]$ reciprocal plane, and shows the same intensity dependence with $h$ and $l$ as the diffuse streaks observed in the $\left[h, 1 \pm q_{b}, l\right]$ planes. For these reasons, one should more likely consider the diffuse scattering as satellite streaks situated at $\pm\left(1-q_{h}\right) b^{*}$ from the above mentioned Bragg reflections.

The half-width at half maximum (H.W.H.M.), $\Delta$, of the diffuse streaks has been measured along the [010] and [102] directions. Their temperature dependence, again identical for both compounds, is given together with the experimental resolution in figure 4. This shows that above $T_{c}, \Delta[102]$ is several times larger than $\Delta[010]$, so that the diffuse streaks are in shape of elongated platelets (or flattened cigars). When $T$ approaches $T_{\mathrm{c}}$ from above, $\Delta$ [102] reaches the experimental resolution with a square root-like law, while $\Delta[010]$ reaches linearly the experimental resolution. Somewhat similar temperature dependence of intrachain and interchain widths of the diffuse scattering have been observed in the 1D organic conductor TMTSF-DMTCNQ [12].

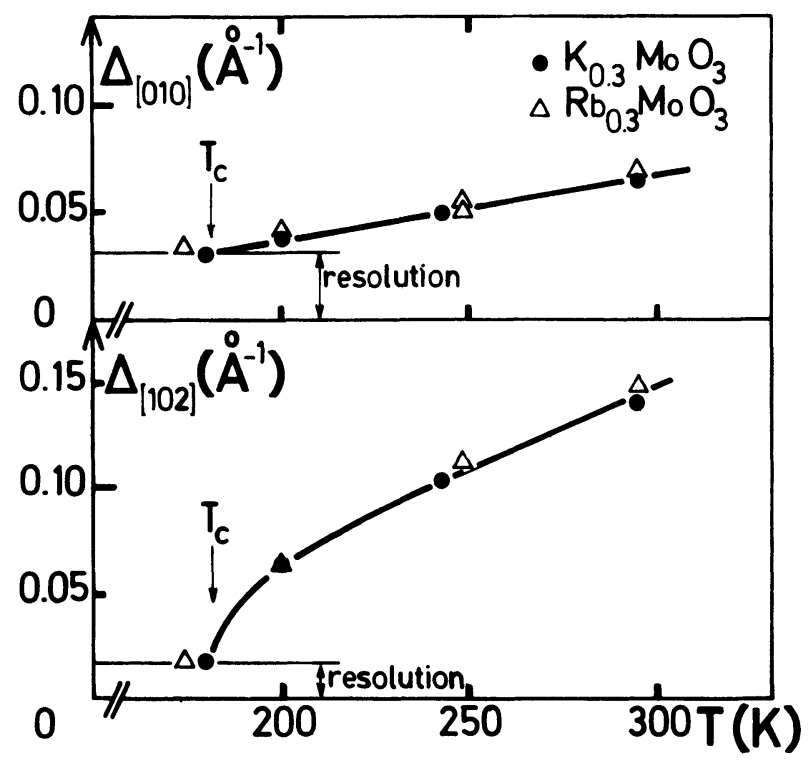

Fig. 4. - Temperature dependence of the half-width at half maximum, $\Delta$, of the diffuse platelets along the [010] and [102] directions for $\mathrm{K}_{0.30} \mathrm{MoO}_{3}$ (solid circles) and $\mathrm{Rb}_{0.30} \mathrm{MoO}_{3}$ (open triangles). The experimental resolution in these two directions is also indicated.

Below $T_{\mathrm{c}} \simeq 180 \mathrm{~K}$, well defined (i.e. resolution limited) reflections are observed (Fig. $2 b$ ). From their position in reciprocal space (Fig. $3 b$ ) they can be considered as the "condensation " of the high temperature diffuse streaks into satellite reflections. Their narrowness shows that they correspond to a long range periodic lattice distortion.

The X-ray pattern of figure $2 b$ shows that in addition to strong satellite reflections at $\pm q_{b} b^{*}$ from layers of Bragg reflections with odd $K$ indices, weak satellite reflections can be observed at $\pm q_{b} b^{*}$ from layers of Bragg reflections with even $K$ indices. These last reflections are announced by streaks of very weak intensity below $200 \mathrm{~K}$. X-ray Weissenberg $\left[h, q_{b}, l\right],\left[h, 1 \pm q_{b}, l\right]$ reciprocal planes locate satellite reflections at a wave vector $\mathbf{q}=\left[0, \pm\left(1-q_{b}\right), 1 / 2\right]$ from the allowed main Bragg reflections (Fig. $3 b$ ). As expected, the satellite intensity is proportional to that of the high 
temperature precursor scattering and has the same wave vector dependence for the two blue bronzes. At $110 \mathrm{~K}$ the incommensurate wave vector component $q_{b}=0.26 \pm 0.01 \mathbf{b}^{*}$ appears to have slightly decreased from its value at R.T.

4. Discussion. - The main result of this study is to show that the blue bronzes undergo a periodic lattice distortion at the same temperature $T_{c}=180 \mathrm{~K}$, where a metal insulator phase transition has been already reported on transport $[4,5]$ and optical [6] properties. This phase transition can also be detected by an anomaly on specific heat data [13] and by a decrease of the magnetic susceptibility [5]. This suggests that a coupling between the lattice and electronic degrees of freedom are responsible for the phase transition. Further proofs will be brought by the analysis of the structural data given below.

The existence of the same critical temperatures for both $\mathrm{K}_{0.30} \mathrm{MoO}_{3}$ and $\mathrm{Rb}_{0.30} \mathrm{MoO}_{3}$, as well as the same temperature dependence of precursor scattering (Fig. 4), show that the alkaline metals are not the driving force of the phase transition. In addition, the same wave vector dependence of the structure factor of the satellite reflections and of the precursor scattering, in spite of the large difference of atomic form factor between $\mathrm{K}$ and $\mathrm{Rb}$, also shows that the alkaline metals are not greatly affected by the phase transition.

Furthermore, it is possible to find, by symmetry considerations, which $\mathrm{MoO}_{6}$ octahedra are mainly involved in the structural distortion. Let us first examine the data obtained at room temperature. The observation of diffuse streaks parallel to the $2 \mathbf{a}^{*}-\mathbf{c}^{*}$ reciprocal direction shows that there is no correlation in atomic displacements between neighbouring (201) sheets (octahedra layers), as expected by the structural anisotropy of the blue bronze. In these sheet directions, the observation of diffuse scattering at $\pm\left(1-q_{b}\right) \mathbf{b}^{*}$ from the allowed main Bragg reflections with even indices (Fig. $3 a$ ) means that the modulation involves entities with $\mathbf{b} / 2$ and $\mathbf{a} / 2+\mathbf{c}$ periodicities. Considering, for the moment, the ideal Mo bronze $\mathrm{K}_{3} \mathrm{Mo}_{10} \mathrm{O}_{30}$ (see the crystal structures described in Figs. $6 c$ and $6 d$ in Ref. [8]), it is easy to reproduce this periodicity by ignoring the $\mathrm{K}$ atoms and $\mathrm{Mo}(1) \mathrm{O}_{6}$ octahedra. The pseudo-unit cell then includes $2 \mathrm{Mo}(2) \mathrm{O}_{6}$ and $2 \mathrm{Mo}(3) \mathrm{O}_{6}$ octahedra, which forms the infinite chains directed along $\mathbf{b}$. The pseudo-symmetry of the diffuse scattering can then be explained if the lattice modulation involves mainly these octahedra. At room temperature when the atomic displacements are small, deviations from the ideal structure lead to secondary effects. Moreover, when the amplitude of the atomic displacements increases, when $T_{\mathrm{c}}$ is approached from above, deviations from the ideal structure, as well as possible distortion of $\mathrm{Mo}(1) \mathrm{O}_{6}$ octahedra may become relevant and break the high temperature pseudosymmetry. But below $200 \mathrm{~K}$ the large difference between the intensities of the satellite reflections around the main Bragg reflections with even $K$ indices and those with odd $K$ indices, still keep the memory of this pseudo-symmetry.

As the $\mathrm{Mo}(2)-\mathrm{O}_{6}$ and $\mathrm{Mo}(3)-\mathrm{O}_{6}$ octahedra are involved in the quasi one-dimensional conduction band, it is natural to propose, by analogy with other $1 \mathrm{D}$ conductors, that the phase transition is Peierls type, as it was already suggested in reference [6]. In 1D conductors, coupling between the $2 k_{\mathrm{F}}$ electronic instability and some special low frequency phonon modes leads to the formation of C.D.W. which fluctuate incoherently from chain to chain, at a phonon frequency above $T_{\mathrm{c}}$, and thus gives rise to X-ray diffuse sheets [7]. Below $T_{\mathrm{c}}$ these C.D.W. become static and couple three-dimensionally, leading to new superlattice periodicities. The structural data obtained with the blue bronze present some difference from those observed on more conventional $1 \mathrm{D}$ conductors like TTF-TCNQ or KCP [7]. They are critically analysed below.

The strongest difference is that precursor effects observed above $T_{\mathrm{c}}$ are not exactly in shape of diffuse sheets often observed in the region of 1D C.D.W. fluctuations. Figure 4 shows that between R.T. and $T_{\mathrm{c}}$ the diffuse scattering appears as elongated platelets with a much larger width in the [102] direction than in the [010] direction. For example at R.T., the H.W.H.M. of the streak gives, after a Lorentzian resolution correction, the following correlation lengths : $\xi_{[010]} \sim 30 \AA$ 
(8 pseudo-unit or octahedra spacing) and $\xi_{[102]} \sim 8 \AA$ (less than 1 pseudo-unit spacing). This shows that at R.T. the C.D.W. fluctuations are well established in the $b$ direction and exhibit weak correlations along [102] and no correlation between $(\overline{201})$ sheets. In this respect structural correlation lengths follow the anisotropy of the electrical resistivity (Fig. 1). Diffuse streaks might be viewed either as a Kohn anomaly associated to a 2D Fermi surface or to the coupling between 1D C.D.W. Observation of a different temperature dependence between $\xi_{[102]}^{-1} \sim \sqrt{T-T_{\mathrm{c}}}($ mean field behaviour) and $\xi_{[010]}^{-1} \sim\left(T-T_{\mathrm{c}}\right.$ ) (quasi one 1D fluctuation regime) seems to accredite the latter possibility. Similar temperature dependence has been reported in the 1D conductor TMTSFDMTCNQ [12] where lateral coupling between C.D.W. fluctuations is observed in a large temperature range above $T_{\mathrm{c}}$, and is predicted by a theoretical treatment of $1 \mathrm{D}$ coupled fluctuations [14].

The Peierls transition is related to the opening of a gap at the Fermi wave vectors $\pm k_{\mathrm{F}}$ of a 1D band structure. This leads to a semiconducting state. The structural data obtained at room temperature, with $1-q_{b}=0.72 b^{*}$ are consistent either with $2 k_{\mathrm{F}}^{\mathrm{I}}=0.72 b^{*}\left(=0.36 \times 2 b^{*}\right.$ if one considers the pseudo-periodicity $b / 2$ discussed previously) or with $2 k_{\mathrm{F}}^{\mathrm{II}}=1.28 b^{*}\left(=0.64 \times 2 b^{*}\right)$. These values should be compared with possible values of $2 k_{\mathrm{F}}$ deduced from the chemical formula and the band structure. Unfortunately, the band structure of $\mathrm{K}_{0.30} \mathrm{MoO}_{3}$ is not accurately known and only speculations can be made at this point. Let us assume in a first approximation that all electrons given by the alkaline metals participate to the conduction band, so that there is 1.5 conduction electron per pseudo-unit cell (pseudo-cluster) of $2 \mathrm{Mo}(2) \mathrm{O}_{6}+2 \mathrm{Mo}(3) \mathrm{O}_{6}$ octahedra. A value of $2 k_{\mathrm{F}}=0.75 b^{*}\left(=0.375 \times 2 b^{*}\right)$ would then correspond to a $1 \mathrm{D}$ conduction band with a degeneracy of 4 (orbital and spin degeneracy of 2). It had been previously proposed that the band structure of the Mo bronzes should be similar to that of $\mathrm{ReO}_{3}$ [6]. But in the blue bronzes, the orbital degeneracy of the $d$ states has been lifted by the low crystal symmetry and the distortion of the $\mathrm{MoO}_{6}$ octahedron, so that the corresponding orbital degeneracy of the hybridized d-p states should be 1 for a $\mathrm{MoO}_{3}$ molecule. Furthermore, in the pseudo-cluster of $4 \mathrm{MoO}_{6}$ octahedra, one expects the formation of molecular orbitals and a further removal of degeneracy, possibly leading to 2 molecular states of lower energy (instead of 4 orbital states) for the pseudo-unit cell, which might be partially occupied by electrons given by the alkaline metals. This would be the case if the splitting of the subbands, due both to the distortion of the relevant $\mathrm{MoO}_{6}$ octahedra and to the existence of the clusters, is large enough to prevent subbands overlap at the Fermi level. The experimental value of $2 k_{\mathrm{F}}^{\mathrm{I}}=0.72 b^{*}$ could be consistent with this picture. One should also notice that the deviation of $2 k_{\mathrm{F}}^{1}$ from the theoretical value of $0.75 b^{*}$ can be accounted for by several mechanisms. First, the stoichiometry of the blue bronze may be slightly different from $\mathrm{K}_{0.30} \mathrm{MoO}_{3}$. Secondly, the number of electrons per pseudo-cluster should be slightly smaller than 1.5 as some d-electron density, found on the Mo(1) sites [9], might belong to localized states not contributing to the conduction band.

Let us now describe the coupling between C.D.W. fluctuations. At room temperature, the observation of diffuse streaks distant of $\pm \cdot\left(1-q_{b}\right) \mathbf{b}^{*}$ from the main Bragg reflections (Fig. $3 a$ ) means that there is in-phase ordering of the modulation of the pseudo-unit cells along the [102] direction. This periodicity is difficult to understand if there is one C.D.W. per pseudo-cluster. In $1 \mathrm{D}$ conductors built with one type of chain only, like KCP, nesting effects on the Fermi surface [15] as well as Coulomb interactions between C.D.W. [16] lead to an antiphase ordering. A suitable explanation for the blue bronzes might be that there is antiphase ordering between 2 C.D.W. per pseudo-cluster. Each C.D.W. might belong to a chain built with entities of two octahedra : $\mathrm{Mo}(2) \mathrm{O}_{6}+\mathrm{Mo}(3) \mathrm{O}_{6}$. This might provide a physical basis for the two-fold orbital degeneracy per pseudo-cluster, suggested above. In addition the strong coupling between these chains might also explain the short range order observed in the [102] direction.

At $T_{\mathrm{c}}$, diffuse streaks « condense " into satellite reflections midway between the main Bragg reflections as shown in figure $3 b$. This ordering corresponds to a doubling of the lattice periodicity 
in the $c$ direction and no change of periodicity along $a$. As the layers of octahedra are parallel to the [102] direction, an antiphase ordering of the sheets of octahedra explains also the lateral periodicities observed below $T_{\mathrm{c}}$. Because of the strong anisotropy of the electron gas, this periodicity can be more likely understood as being that minimizing the Coulomb interaction between the C.D.W. belonging to neighbouring sheets.

A specific feature of the diffuse scattering of the blue bronzes, compared to that of TTF-TCNQ and KCP [7], is that the diffuse streaks appear in Brillouin zones where a thermal diffuse scattering in broad sheets perpendicular to the [102] direction is observed (see Fig. 2a). This might indicate that the Kohn anomaly takes place in a valley of softer phonons. (The thermal scattering observed around strong Bragg reflections is probably the signature of softer phonons with acoustic-like displacements). It is thus tempting to suggest that, in the blue bronzes, the Kohn anomaly is forced to develop in a pre-existing valley of low frequency phonons, where there is a strong electron phonon coupling as a consequence of larger atomic displacements. Inelastic neutron scattering experiments are now necessary to corroborate the picture.

Acknowledgments. - The authors wish to thank E. Bervas for the electrical resistivity measurements, J. Dumas and C. Filippini for extremely helpful discussions and M. Marezio for stimulating comments.

\section{References}

[1] Hagenmuller, P., Prog. Solid Stute Chem. 5 (1972) 71.

[2] Bouchard Jr., G. H., Perlstein, J. and Sienko, M. J., Inorg. Chem. 6 (1967) 1682.

[3] Buder, R., Devenyi, J., Dumas, J., Marcus, J., Mercier, J. and Schlenker, C., J. Physique Lett. 43 (1982) L-59.

[4] Fogle, W. and Perlstein, J. M., Phys. Rev. B 6 (1972) 1402.

[5] Brusetti, R., Chakraverty, B. K., Devenyi, J., Dumas, J., Marcus, J. and Schlenker, C., Recent Developments in Cond. Matter Phys., Ed. J. T. Devreese et al. (Plenum) Vol. 2, 1982, p. 181.

Buder, R., Dumas, J., Marcus, J., Mercier, J. and Schlenker, C., Congrès de la Soc. Française de Phys., Bulletin des Résumés, Clermont-Ferrand 1981, p. 204.

[6] Travaglini, G., Wachter, P., Marcus, J. and Schlenker, C., Solid State Commun. 37 (1981) 599.

[7] See for example Highly Conducting One Dimensional Solids, edited by J. T. Devreese et al. (Plenum) 1979.

[8] Graham, J. and Wadsley, A. D., Acta Cryst allogr. 20 (1966) 93.

[9] Chenavas, J., Ghedira, M. and Marezio, M., Private comm. and to be published. See also Bull. Amer. Phys. Soc. 26 (1981) 449.

[10] Strobel, P. and Greenblatt, M., J. Solid Stute Chem. 36 (1981) 331.

[11] Cooper, J. R., Jerome, D., Etemad, S. and Engler, E. M., Solid State Commun. 22 (1977) 257.

[12] Pouget, J. P., Chemicu Scriptu 17 (1981) 85.

Forro, L., Zuppiroli, L., Pouget, J. P. and Bechgaard, K., Phys. Rev. B (in press).

[13] Dumas, J., Filippini, C., Marcus, J. and Schlenker, C. (to be published).

[14] See for example Dieterich, W., Adv. Phys. 25 (1976) 615.

[15] Horowitz, B., Gutfreund, M. and Weger, M., Phys. Rev. B 12 (1975) 3174.

[16] Saub, K., Barisic, S. and Friedel, J., Phys. Lett. 56A (1976) 302. 